\title{
12. VELOCITIES AND ELASTIC MODULI OF VOLCANIC AND SEDIMENTARY ROCKS RECOVERED ON DSDP LEG 25
}

\author{
N. I. Christensen, D. M. Fountain, R. L. Carlson, and M. H. Salisbury, \\ Department of Geological Sciences, University of Washington, Seattle, Washington
}

\section{INTRODUCTION}

On Leg 25 of the Deep Sea Drilling Project five holes penetrated basement, and a total of approximately 12.7 meters of basalt were recovered. We have measured compressional and shear wave velocities to hydrostatic pressures of $6 \mathrm{~kb}$ in representative samples of basalt from each hole and a relatively high density limestone from Site 249. In addition, compressional wave velocities are reported for two sedimentary rocks recovered from Sites 246 and 249 .

The three basalts studied from Site 249 have intersertal textures with altered feldspar laths and groundmasses highly weathered to greenish yellow nontronite(?) and brown iddingsite. Weathering appears to decrease slightly with depth, and Sample 249-33-2, 126-129 cm is highly vesicular.

Samples 239-21-1, 46-49 $\mathrm{cm}$ and 239-20-1, $125-128 \mathrm{~cm}$ are intersertal to felty textured basalts with some alteration of their groundmasses. Basalt Sample 240-7-1, 120-123 cm is similar to the basalts from Site 239 in both texture and alteration.

Sample $245-19-1,37-40 \mathrm{~cm}$ is a coarse-grained, relatively fresh basalt with an intergranular texture. Alteration is confined to pyroxenes in the groundmass. The two basalts from Site 248 have intersertal textures and contain large (up to $0.7 \mathrm{~cm}$ in length) feldspar and subordinate pyroxene phenocrysts. The deeper of the two samples (248-17-2, $122-125 \mathrm{~cm}$ ) has less alteration and is slightly coarser grained than Sample 248-15-1, 35-38 cm.

Sample $249-27-2,133-136 \mathrm{~cm}$ is a slightly recrystallized biomicrite. Sample $249-28-2,56-59 \mathrm{~cm}$ is a glauconitebearing mudstone, and Sample $246-10, \mathrm{CC}(3 \mathrm{~cm})$ is a volcanic sand cemented with carbonate.

\section{VELOCITIES AND ELASTIC MODULI}

Velocities and bulk densities calculated from the weights and dimensions of the samples are given in Table 1. The experimental technique used to obtain the velocities has been described by Birch (1960). Samples were water saturated prior to the measurements and during the runs, pore pressures were maintained at values much less than external pressure by placing 100-mesh screens between the samples and copper jackets. For all samples included in the present study, wave propagation directions were parallel to the drill hole axes.

Ratios of compressional to shear velocities $\left(V_{p} / V_{s}\right)$, Poisson's ratios $(\sigma)$, seismic parameters $(\phi)$, bulk moduli $(K)$, compressibilities $(\beta)$, shear moduli $(\mu)$, Young's moduli $(E)$, and Lamé's constant $(\lambda)$ calculated from densities and velocities are given in Table 2 for selected pressures.

\section{VELOCITY-DENSITY RELATIONS}

Densities for the nine basalt samples from Leg 25 range from a low value of $2.188 \mathrm{~g} / \mathrm{cc}$ to a maximum of 2.889 $\mathrm{g} / \mathrm{cc}$, while the range in compressional wave velocities is 3.61 to $6.11 \mathrm{~km} / \mathrm{sec}$. Similarly, the shear wave velocities range from a minimum of $1.96 \mathrm{~km} / \mathrm{sec}$ to a maximum of $3.30 \mathrm{~km} / \mathrm{sec}$. The wide variation in densities of these rocks is primarily due to weathering. The basalt of least bulk density $(249-33-2,126-129 \mathrm{~cm})$ is, however, quite vesicular.

Christensen and Salisbury (1972; in press) demonstrated the existence of a strong correlation between density and velocity among oceanic basalts. Figures 1 and 2 show plots of compressional and shear wave velocity against density for nine DSDP Leg 25 basalts. For comparison, 45 Atlantic and Pacific basalts (Christensen and Salisbury, in press) are also shown. These velocities correspond to $0.5 \mathrm{~kb}$ pressure, approximately the pressure appropriate to oceanic layer 2 .

Also included in the figures are least-squares regression lines for 32 Pacific samples studied by Christensen and Salisbury (in press), with the following line parameters:

$$
\begin{aligned}
& V_{p}=3.32 \rho-3.55 \mathrm{~km} / \mathrm{sec} \\
& V_{S}=2.15 \rho-3.04 \mathrm{~km} / \mathrm{sec}
\end{aligned}
$$

Christensen and Salisbury (in press) observed that velocities are correctly predicted from these equations to within $\pm 0.2 \mathrm{~km} / \mathrm{sec}$. Although it is apparent from the data point distribution that a parabolic fit may be more accurate, the accuracy of the linear equations is unchanged for densities greater than about $2.3 \mathrm{~g} / \mathrm{cc}$.

\section{DISCUSSION}

Several investigations of elastic wave velocities in igneous and metamorphic rocks have emphasized the importance of water saturation on compressional wave velocities (e.g., Wyllie et al., 1958; Nur and Simmons, 1969; Christensen, 1970 ). It has generally been found that at pressures below a few kilobars compressional wave velocities are significantly higher in water-saturated rocks than in unsaturated rocks, whereas shear wave velocities are little affected. For DSDP basalts and sedimentary rocks the differences in saturated and unsaturated velocities vary considerably from one sample to another. This variation is most likely related to differences in porosity and pore geometry of the samples (Christensen et al., in press). An example of the influence of water saturation on compressional wave velocities for a basalt included in the present study is illustrated in Figure 3 , which represents two sets of measurements made on the 
TABLE 1

Compressional (P) and Shear (S) Wave Velocities

\begin{tabular}{|c|c|c|c|c|c|c|c|c|c|c|}
\hline \multirow[b]{2}{*}{ Sample } & \multirow{2}{*}{$\begin{array}{l}\text { Bulk } \\
\text { Density }\end{array}$} & \multirow[b]{2}{*}{ Mode } & \multicolumn{8}{|c|}{ Velocity $(\mathrm{km} / \mathrm{sec})$ at Varying Pressures $(\mathrm{kb})$} \\
\hline & & & 0.2 & 0.4 & 0.6 & 0.8 & 1.0 & 2.0 & 4.0 & 6.0 \\
\hline $\begin{array}{l}239-20-1, \\
125-128 \mathrm{~cm}\end{array}$ & $\begin{array}{l}2.723 \\
2.723\end{array}$ & $\begin{array}{l}\mathrm{P} \\
\mathrm{S}\end{array}$ & $\begin{array}{l}5.39 \\
2.95\end{array}$ & $\begin{array}{l}5.42 \\
2.96\end{array}$ & $\begin{array}{l}5.44 \\
2.97\end{array}$ & $\begin{array}{l}5.46 \\
2.97\end{array}$ & $\begin{array}{l}5.47 \\
2.98\end{array}$ & $\begin{array}{l}5.50 \\
2.99\end{array}$ & $\begin{array}{l}5.57 \\
3.00\end{array}$ & $\begin{array}{l}5.63 \\
3.02\end{array}$ \\
\hline $\begin{array}{l}239-21-1 \\
46-49 \mathrm{~cm}\end{array}$ & $\begin{array}{l}2.762 \\
2.762\end{array}$ & $\begin{array}{l}\mathrm{P} \\
\mathrm{S}\end{array}$ & $\begin{array}{l}5.43 \\
2.98\end{array}$ & $\begin{array}{l}5.46 \\
2.99\end{array}$ & $\begin{array}{l}5.48 \\
2.99\end{array}$ & $\begin{array}{l}5.49 \\
3.00\end{array}$ & $\begin{array}{l}5.50 \\
3.00\end{array}$ & $\begin{array}{l}5.54 \\
3.01\end{array}$ & $\begin{array}{l}5.60 \\
3.03\end{array}$ & $\begin{array}{l}5.66 \\
3.05\end{array}$ \\
\hline $\begin{array}{l}240-7-1, \\
120-123 \mathrm{~cm}\end{array}$ & $\begin{array}{l}2.795 \\
2.795\end{array}$ & $\begin{array}{l}\mathrm{P} \\
\mathrm{S}\end{array}$ & $\begin{array}{l}5.84 \\
3.24\end{array}$ & $\begin{array}{l}5.86 \\
3.25\end{array}$ & $\begin{array}{l}5.87 \\
3.26\end{array}$ & $\begin{array}{l}5.89 \\
3.26\end{array}$ & $\begin{array}{l}5.89 \\
3.27\end{array}$ & $\begin{array}{l}5.93 \\
3.28\end{array}$ & $\begin{array}{l}6.00 \\
3.30\end{array}$ & $\begin{array}{l}6.07 \\
3.32\end{array}$ \\
\hline $\begin{array}{l}245-19-1, \\
37-40 \mathrm{~cm}\end{array}$ & $\begin{array}{l}2.889 \\
2.889\end{array}$ & $\begin{array}{l}\mathrm{P} \\
\mathrm{S}\end{array}$ & $\begin{array}{l}6.07 \\
3.29\end{array}$ & $\begin{array}{l}6.09 \\
3.29\end{array}$ & $\begin{array}{l}6.12 \\
3.30\end{array}$ & $\begin{array}{l}6.14 \\
3.31\end{array}$ & $\begin{array}{l}6.16 \\
3.32\end{array}$ & $\begin{array}{l}6.21 \\
3.34\end{array}$ & $\begin{array}{l}6.28 \\
3.37\end{array}$ & $\begin{array}{l}6.35 \\
3.39\end{array}$ \\
\hline $\begin{array}{l}246-10 \mathrm{CC}, \\
(3 \mathrm{~cm}) \\
\text { Sedimentary }\end{array}$ & 2.095 & $\mathbf{P}$ & 3.74 & 3.83 & 3.89 & 3.94 & 3.98 & 4.12 & 4.32 & 4.51 \\
\hline $\begin{array}{l}248-15-1, \\
35-38 \mathrm{~cm}\end{array}$ & $\begin{array}{l}2.676 \\
2.676\end{array}$ & $\begin{array}{l}P \\
S\end{array}$ & $\begin{array}{l}5.35 \\
2.89\end{array}$ & $\begin{array}{l}5.38 \\
2.90\end{array}$ & $\begin{array}{l}5.40 \\
2.92\end{array}$ & $\begin{array}{l}5.42 \\
2.93\end{array}$ & $\begin{array}{l}5.44 \\
2.94\end{array}$ & $\begin{array}{l}5.49 \\
2.96\end{array}$ & $\begin{array}{l}5.60 \\
2.98\end{array}$ & $\begin{array}{l}5.70 \\
3.00\end{array}$ \\
\hline $\begin{array}{l}248-17-2 \\
122-125 \mathrm{~cm}\end{array}$ & $\begin{array}{l}2.758 \\
2.758\end{array}$ & $\begin{array}{l}P \\
\text { S }\end{array}$ & $\begin{array}{l}5.80 \\
3.19\end{array}$ & $\begin{array}{l}5.86 \\
3.22\end{array}$ & $\begin{array}{l}5.89 \\
3.23\end{array}$ & $\begin{array}{l}5.92 \\
3.24\end{array}$ & $\begin{array}{l}5.93 \\
3.25\end{array}$ & $\begin{array}{l}5.99 \\
3.28\end{array}$ & $\begin{array}{l}6.06 \\
3.32\end{array}$ & $\begin{array}{l}6.13 \\
3.34\end{array}$ \\
\hline $\begin{array}{l}249-27-2, \\
133-136 \mathrm{~cm} \\
\text { (Sedimentary) }\end{array}$ & $\begin{array}{l}2.250 \\
2.250\end{array}$ & $\begin{array}{l}P \\
S\end{array}$ & $\begin{array}{l}4.28 \\
2.36\end{array}$ & $\begin{array}{l}4.40 \\
2.50\end{array}$ & $\begin{array}{l}4.47 \\
2.59\end{array}$ & $\begin{array}{l}4.53 \\
2.64\end{array}$ & $\begin{array}{l}4.58 \\
2.69\end{array}$ & $\begin{array}{l}4.80 \\
2.83\end{array}$ & $\begin{array}{l}5.07 \\
2.98\end{array}$ & $\begin{array}{l}5.20 \\
3.04\end{array}$ \\
\hline $\begin{array}{l}249-28-2 \text {, } \\
56-59 \mathrm{~cm} \\
\text { (Sedimentary) }\end{array}$ & 1.491 & $\mathbf{P}$ & 2.08 & 2.14 & 2.17 & 2.21 & 2.24 & 2.38 & 2.64 & 2.89 \\
\hline $\begin{array}{l}249-33-2 \\
27-30 \mathrm{~cm}\end{array}$ & $\begin{array}{l}2.321 \\
2.321\end{array}$ & $\begin{array}{l}\mathrm{P} \\
\mathrm{S}\end{array}$ & 3.53 & 3.59 & 3.63 & $\begin{array}{l}3.67 \\
2.17\end{array}$ & $\begin{array}{l}3.70 \\
2.19\end{array}$ & $\begin{array}{l}3.84 \\
2.27\end{array}$ & $\begin{array}{l}4.03 \\
2.39\end{array}$ & $\begin{array}{l}4.21 \\
2.49\end{array}$ \\
\hline $\begin{array}{l}249-33-2 \\
126-129 \mathrm{~cm}\end{array}$ & $\begin{array}{l}2.188 \\
2.188\end{array}$ & $\begin{array}{l}P \\
S\end{array}$ & $\begin{array}{l}3.81 \\
1.94\end{array}$ & $\begin{array}{l}3.83 \\
1.96\end{array}$ & $\begin{array}{l}3.85 \\
1.97\end{array}$ & $\begin{array}{l}3.86 \\
1.98\end{array}$ & $\begin{array}{l}3.88 \\
1.99\end{array}$ & $\begin{array}{l}3.97 \\
2.05\end{array}$ & $\begin{array}{l}4.14 \\
2.12\end{array}$ & $\begin{array}{l}4.31 \\
2.16\end{array}$ \\
\hline $\begin{array}{l}249-33-3 \\
128-131 \mathrm{~cm}\end{array}$ & $\begin{array}{l}2.393 \\
2.393\end{array}$ & $\begin{array}{l}\mathrm{P} \\
\mathrm{S}\end{array}$ & $\begin{array}{l}4.17 \\
2.17\end{array}$ & $\begin{array}{l}4.21 \\
2.20\end{array}$ & $\begin{array}{l}4.25 \\
2.25\end{array}$ & $\begin{array}{l}4.28 \\
2.28\end{array}$ & $\begin{array}{l}4.30 \\
2.30\end{array}$ & $\begin{array}{l}4.39 \\
2.38\end{array}$ & $\begin{array}{l}4.54 \\
2.44\end{array}$ & $\begin{array}{l}4.68 \\
2.47\end{array}$ \\
\hline
\end{tabular}

same sample. Measurements were made first with the rock in a saturated condition and again after the core had been air dried for several days.

Based on seismic velocities of DSDP sedimentary rocks from Leg 19, Christensen et al. (in press) suggested that high velocity sedimentary rocks may overlie oceanic basalts of lower velocity, creating a low velocity zone in some parts of the uppermost oceanic crust. The possibility for such a condition is illustrated by the data presented here for Site 249 , where a limestone with a relatively high velocity (4.43 $\mathrm{km} / \mathrm{sec}$ at $0.5 \mathrm{~kb}$ ) overlies a low velocity mudstone $(2.15$ $\mathrm{km} / \mathrm{sec}$ ). Both sedimentary units are found above low velocity basalts (3.61 to $4.23 \mathrm{~km} / \mathrm{sec}$ ), thus creating a low velocity layer in the uppermost crust in the vicinity of Site 249.

The low velocities and densities of the basalts from Site 249 are related to submarine weathering, as discussed by Christensen and Salisbury (1972; in press). A diffuse but significant trend of decreasing density of DSDP basalts with age can be seen in Figure 4, in which the densities of both intrusive and extrusive submarine basalts from previous measurements and from Leg 25 are plotted against age. The geophysical significance of this trend, ascribed to progressive submarine weathering, is two-fold: (1) the velocitydensity relationships of Figures 1 and 2 prescribe that where such weathering effects extend to sufficient depth, seismic refraction velocities in layer 2 should decrease with age (Christensen and Salisbury, 1972; in press); and (2) where high velocity sediments such as the limestone from Site 249 are found over low density basalts, the uppermost levels of layer 2 will behave as a thin low velocity zone; such zones will be particularly abundant in areas of old oceanic crust. Whether these low velocity zones can be detected by seismic refraction techniques depends upon the thicknesses and velocity contrasts of the various rock units.

\section{ACKNOWLEDGMENTS}

We wish to thank Robert McConaghy for maintaining the high pressure system. This investigation was supported by the Office of Naval Research contract N-00014-67-A0103-0014 and National Science Foundation grant GA-36138.

\section{REFERENCES}

Birch, F., 1960. The velocity of compressional waves in rocks to 10 kilobars, I: J. Geophys. Res., v. 65, p. 1083.

Christensen, N. I., 1970. Compressional wave velocities in basalts from the Juan de Fuca Ridge: J. Geophys. Res., v. 75 , p. 2773 .

Christensen, N. I., Fountain, D. M., and Stewart, R. J., in press. Oceanic crustal basement: A comparison of seismic properties of DSDP basalts and consolidated sediments: Marine Geol.

Christensen, N. I. and Salisbury, M. H., 1972. Sea floor spreading, progressive alteration of layer 2 basalts, and associated changes in seismic velocities: Earth Planet. Sci. Lett., v. 15 , p. 367. 
TABLE 2

Elastic Constants, Leg 25

\begin{tabular}{|c|c|c|c|c|c|c|c|c|c|}
\hline Sample & $\begin{array}{c}\text { Pressure } \\
(\mathrm{kb})\end{array}$ & $V_{p} / V_{s}$ & $\sigma$ & $\begin{array}{c}\Phi \\
\left(\mathrm{km} / \sec ^{-1}\right)^{2} \\
\end{array}$ & $\begin{array}{c}K \\
(\mathrm{Mb})\end{array}$ & $\begin{array}{c}\beta \\
\left(\mathrm{Mb}^{-1}\right)\end{array}$ & $\begin{array}{c}\mu \\
(\mathrm{Mb})\end{array}$ & $\begin{array}{c}E \\
(\mathrm{Mb})\end{array}$ & $\begin{array}{c}\lambda \\
(\mathrm{Mb})\end{array}$ \\
\hline $\begin{array}{l}239-20-1 \\
125-128 \mathrm{~cm}\end{array}$ & $\begin{array}{l}0.4 . \\
1.0 \\
2.0 \\
6.0\end{array}$ & $\begin{array}{l}1.83 \\
1.84 \\
1.84 \\
1.86\end{array}$ & $\begin{array}{l}0.29 \\
0.29 \\
0.29 \\
0.30\end{array}$ & $\begin{array}{l}17.72 \\
18.06 \\
18.35 \\
19.41\end{array}$ & $\begin{array}{l}0.48 \\
0.49 \\
0.50 \\
0.53\end{array}$ & $\begin{array}{l}2.07 \\
2.03 \\
1.99 \\
1.87\end{array}$ & $\begin{array}{l}0.24 \\
0.24 \\
0.24 \\
0.25\end{array}$ & $\begin{array}{l}0.61 \\
0.62 \\
0.63 \\
0.65\end{array}$ & $\begin{array}{l}0.32 \\
0.33 \\
0.34 \\
0.37\end{array}$ \\
\hline $\begin{array}{l}239-21-1 \\
46-49 \mathrm{~cm}\end{array}$ & $\begin{array}{l}0.4 \\
1.0 \\
2.0 \\
6.0\end{array}$ & $\begin{array}{l}1.83 \\
1.83 \\
1.84 \\
1.86\end{array}$ & $\begin{array}{l}0.29 \\
0.29 \\
0.29 \\
0.30\end{array}$ & $\begin{array}{l}17.91 \\
18.27 \\
18.51 \\
19.51\end{array}$ & $\begin{array}{l}0.49 \\
0.51 \\
0.51 \\
0.54\end{array}$ & $\begin{array}{l}2.02 \\
1.98 \\
1.95 \\
1.84\end{array}$ & $\begin{array}{l}0.25 \\
0.25 \\
0.25 \\
0.26\end{array}$ & $\begin{array}{l}0.63 \\
0.64 \\
0.65 \\
0.67\end{array}$ & $\begin{array}{l}0.33 \\
0.34 \\
0.35 \\
0.37\end{array}$ \\
\hline $\begin{array}{l}240-7-1, \\
120-123 \mathrm{~cm}\end{array}$ & $\begin{array}{l}0.4 \\
1.0 \\
2.0 \\
6.0\end{array}$ & $\begin{array}{l}1.80 \\
1.80 \\
1.81 \\
1.83\end{array}$ & $\begin{array}{l}0.28 \\
0.28 \\
0.28 \\
0.29\end{array}$ & $\begin{array}{l}20.27 \\
20.48 \\
20.74 \\
20.01\end{array}$ & $\begin{array}{l}0.57 \\
0.57 \\
0.58 \\
0.62\end{array}$ & $\begin{array}{l}1.76 \\
1.74 \\
1.72 \\
1.61\end{array}$ & $\begin{array}{l}0.29 \\
0.30 \\
0.30 \\
0.31\end{array}$ & $\begin{array}{l}0.75 \\
0.76 \\
0.77 \\
0.79\end{array}$ & $\begin{array}{l}0.37 \\
0.37 \\
0.38 \\
0.42\end{array}$ \\
\hline $\begin{array}{l}245-19-1 \\
37-40 \mathrm{~cm}\end{array}$ & $\begin{array}{l}0.4 \\
1.0 \\
2.0 \\
6.0\end{array}$ & $\begin{array}{l}1.85 \\
1.86 \\
1.86 \\
1.87\end{array}$ & $\begin{array}{l}0.29 \\
0.30 \\
0.30 \\
0.30\end{array}$ & $\begin{array}{l}22.66 \\
23.21 \\
23.59 \\
24.89\end{array}$ & $\begin{array}{l}0.66 \\
0.67 \\
0.68 \\
0.73\end{array}$ & $\begin{array}{l}1.53 \\
1.49 \\
1.46 \\
1.38\end{array}$ & $\begin{array}{l}0.31 \\
0.32 \\
0.32 \\
0.33\end{array}$ & $\begin{array}{l}0.81 \\
0.82 \\
0.84 \\
0.86\end{array}$ & $\begin{array}{l}0.45 \\
0.46 \\
0.47 \\
0.50\end{array}$ \\
\hline $\begin{array}{l}248-15-1, \\
35-38 \mathrm{~cm}\end{array}$ & $\begin{array}{l}0.4 \\
1.0 \\
2.0 \\
6.0\end{array}$ & $\begin{array}{l}1.85 \\
1.85 \\
1.85 \\
1.90\end{array}$ & $\begin{array}{l}0.29 \\
0.29 \\
0.29 \\
0.31\end{array}$ & $\begin{array}{l}17.65 \\
18.02 \\
18.38 \\
20.34\end{array}$ & $\begin{array}{l}0.47 \\
0.48 \\
0.49 \\
0.55\end{array}$ & $\begin{array}{l}2.11 \\
2.07 \\
2.02 \\
1.82\end{array}$ & $\begin{array}{l}0.23 \\
0.23 \\
0.24 \\
0.24\end{array}$ & $\begin{array}{l}0.58 \\
0.60 \\
0.61 \\
0.63\end{array}$ & $\begin{array}{l}0.32 \\
0.33 \\
0.34 \\
0.39\end{array}$ \\
\hline $\begin{array}{l}248-17-2, \\
122-125 \mathrm{~cm}\end{array}$ & $\begin{array}{l}0.4 \\
1.0 \\
2.0 \\
6.0\end{array}$ & $\begin{array}{l}1.82 \\
1.82 \\
1.82 \\
1.84\end{array}$ & $\begin{array}{l}0.28 \\
0.28 \\
0.29 \\
0.29\end{array}$ & $\begin{array}{l}20.48 \\
21.07 \\
21.43 \\
22.58\end{array}$ & $\begin{array}{l}0.56 \\
0.58 \\
0.59 \\
0.63\end{array}$ & $\begin{array}{l}1.77 \\
1.72 \\
1.69 \\
1.59\end{array}$ & $\begin{array}{l}0.29 \\
0.29 \\
0.30 \\
0.31\end{array}$ & $\begin{array}{l}0.73 \\
0.75 \\
0.76 \\
0.79\end{array}$ & $\begin{array}{l}0.38 \\
0.39 \\
0.39 \\
0.42\end{array}$ \\
\hline $\begin{array}{l}249-27-2, \\
133-136 \mathrm{~cm} \\
\text { (Sedimentary) }\end{array}$ & $\begin{array}{l}0.4 \\
1.0 \\
2.0 \\
6.0\end{array}$ & $\begin{array}{l}1.76 \\
1.70 \\
1.70 \\
1.71\end{array}$ & $\begin{array}{l}0.26 \\
0.24 \\
0.23 \\
0.24\end{array}$ & $\begin{array}{l}10.94 \\
11.32 \\
12.33 \\
14.48\end{array}$ & $\begin{array}{l}0.25 \\
0.26 \\
0.28 \\
0.33\end{array}$ & $\begin{array}{l}4.05 \\
3.91 \\
3.58 \\
3.01\end{array}$ & $\begin{array}{l}0.14 \\
0.16 \\
0.18 \\
0.21\end{array}$ & $\begin{array}{l}0.36 \\
0.40 \\
0.45 \\
0.52\end{array}$ & $\begin{array}{l}0.15 \\
0.15 \\
0.16 \\
0.19\end{array}$ \\
\hline $\begin{array}{l}249-33-2, \\
27-30 \mathrm{~cm}\end{array}$ & $\begin{array}{l}0.4 \\
1.0 \\
2.0 \\
6.0\end{array}$ & $\begin{array}{l}-- \\
1.69 \\
1.69 \\
1.69\end{array}$ & $\begin{array}{l}-\overline{0.23} \\
0.23 \\
0.23\end{array}$ & $\begin{array}{l}\overline{7.28} \\
7.82 \\
9.33\end{array}$ & $\begin{array}{l}\overrightarrow{0.17} \\
0.18 \\
0.22\end{array}$ & $\begin{array}{l}-- \\
5.89 \\
5.45 \\
4.49\end{array}$ & $\begin{array}{l}-\overline{0.11} \\
0.12 \\
0.15\end{array}$ & $\begin{array}{l}-\overline{0.27} \\
0.30 \\
0.36\end{array}$ & $\begin{array}{l}-\overline{0.10} \\
0.10 \\
0.13\end{array}$ \\
\hline $\begin{array}{l}249-33-2, \\
126-129 \mathrm{~cm}\end{array}$ & $\begin{array}{l}0.4 \\
1.0 \\
2.0 \\
6.0\end{array}$ & $\begin{array}{l}1.96 \\
1.94 \\
1.94 \\
2.00\end{array}$ & $\begin{array}{l}0.32 \\
0.32 \\
0.32 \\
0.33\end{array}$ & $\begin{array}{r}9.53 \\
9.71 \\
10.08 \\
12.20\end{array}$ & $\begin{array}{l}0.21 \\
0.21 \\
0.22 \\
0.27\end{array}$ & $\begin{array}{l}4.78 \\
4.69 \\
4.49 \\
3.66\end{array}$ & $\begin{array}{l}0.08 \\
0.09 \\
0.09 \\
0.10\end{array}$ & $\begin{array}{l}0.22 \\
0.23 \\
0.24 \\
0.27\end{array}$ & $\begin{array}{l}0.15 \\
0.15 \\
0.16 \\
0.20\end{array}$ \\
\hline $\begin{array}{l}249-33-3, \\
128-131 \mathrm{~cm}\end{array}$ & $\begin{array}{l}0.4 \\
1.0 \\
2.0 \\
6.0\end{array}$ & $\begin{array}{l}1.91 \\
1.87 \\
1.85 \\
1.89\end{array}$ & $\begin{array}{l}0.31 \\
0.30 \\
0.29 \\
0.31\end{array}$ & $\begin{array}{l}11.27 \\
11.41 \\
11.72 \\
13.53\end{array}$ & $\begin{array}{l}0.27 \\
0.27 \\
0.28 \\
0.33\end{array}$ & $\begin{array}{l}3.70 \\
3.65 \\
3.54 \\
3.03\end{array}$ & $\begin{array}{l}0.12 \\
0.13 \\
0.14 \\
0.15\end{array}$ & $\begin{array}{l}0.31 \\
0.33 \\
0.35 \\
0.38\end{array}$ & $\begin{array}{l}0.19 \\
0.19 \\
0.19 \\
0.23\end{array}$ \\
\hline
\end{tabular}

, in press. Velocities, elastic moduli and weathering-age relations for Pacific Layer 2 basalts: Earth Planet. Sci. Lett.

Fox, P. J., Schreiber, E., and Peterson, J., 1972. Compressional wave velocities in basalt and altered basalt recovered during Leg 14. In Hayes, D. E., Pimm, A. C., et al., Initial Reports of the Deep Sea Drilling Project, Volume XIV: Washington (U.S. Government Printing Office), p. 773.
Nur, A. and Simmons, G., 1969. The effect of saturation on velocity in low porosity rocks: Earth Planet. Sci. Lett., v. 7 , p. 183 .

Wyllie, M. R. J., Gregory, A. R., and Gardner, G. G. F., 1958. An experimental investigation of factors affecting elastic wave velocities in porous media: Geophysics, v. 23 , p. 459. 


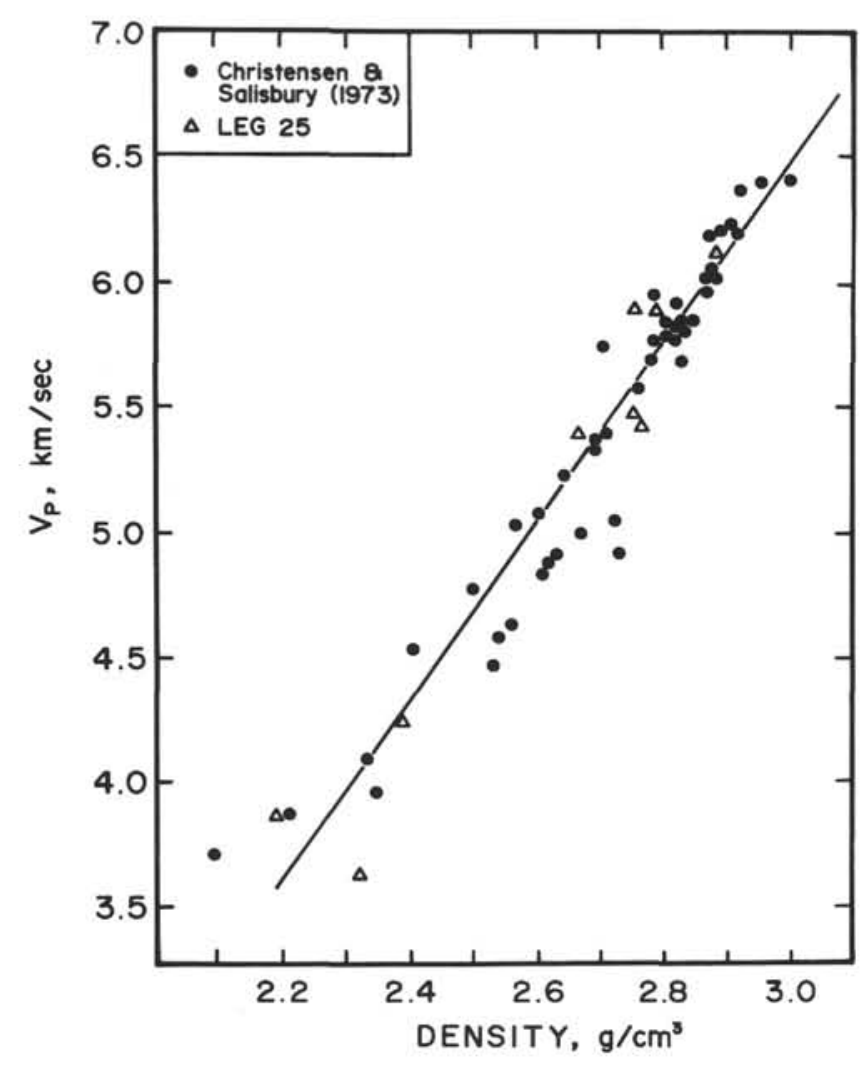

Figure 1. Compressional wave velocities versus densities at $0.5 \mathrm{~kb}$.

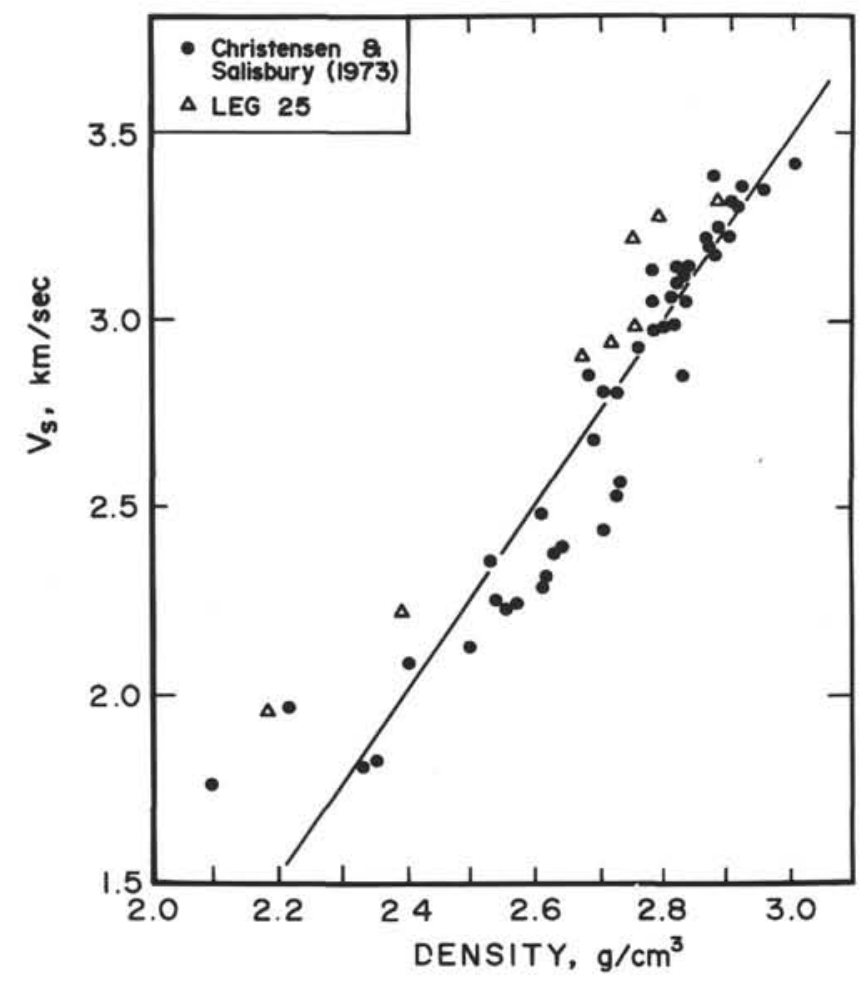

Figure 2. Shear wave velocities versus densities at $0.5 \mathrm{~kb}$.

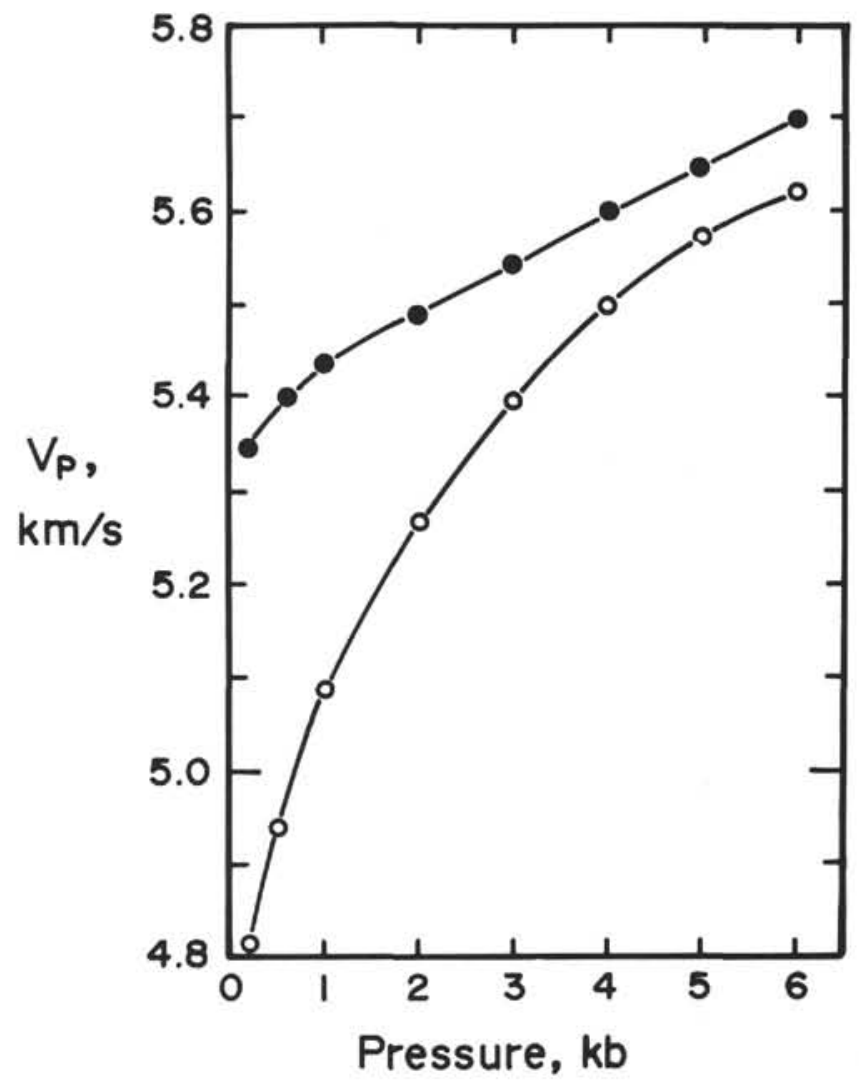

Figure 3. Velocities for basalt 25-248-15-1, 35-38 cm, water-saturated (open circles) and air dry (solid circles).

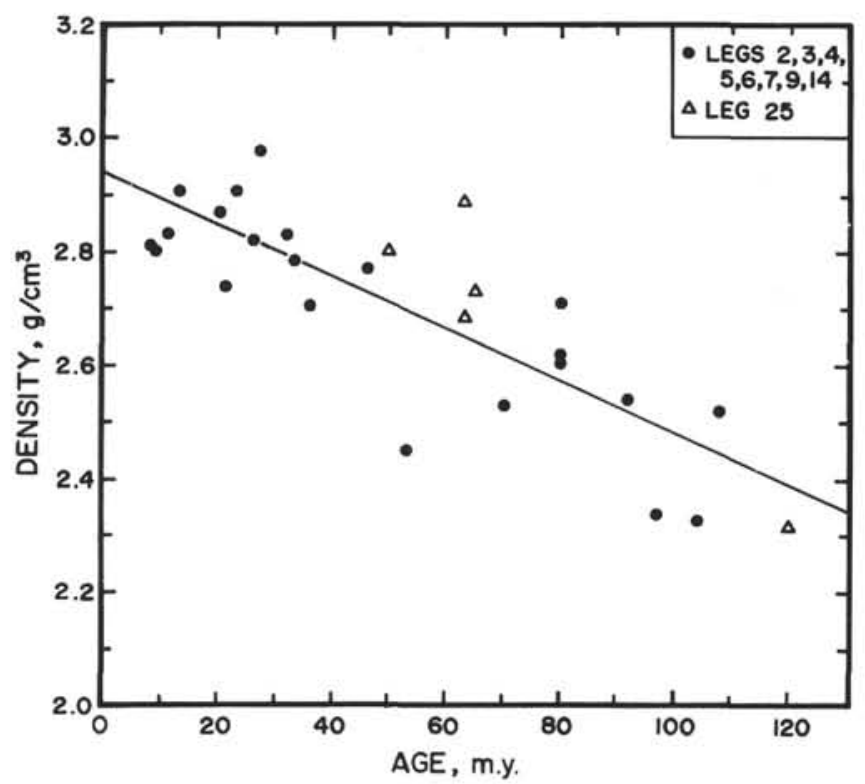

Figure 4. Density-age relations for layer 2 basalts. Data are from Christensen and Salisbury (1972, 1973), Fox et al. (1972) and Table 1. 